\title{
Introduction to the thematic issue
}

\author{
Davy Preuveneers ${ }^{\mathrm{a},{ }^{*}}$, Paulo Novais ${ }^{\mathrm{b}}$ and Juan M. Corchado ${ }^{\mathrm{c}}$ \\ a IBBT-DistriNet, KU Leuven, Celestijnenlaan 200A, B-3001 Heverlee, Belgium \\ ${ }^{\mathrm{b}}$ Deparment of Informatics, University of Minho, Campus de Gualtar, 4710-057 Braga, Portugal \\ ${ }^{c}$ Facultad de Ciencias, Universidad de Salamanca, Plaza de la Merced s/n, Salamanca 37008, Spain
}

\section{Introduction}

This Thematic Issue focuses on software engineering perspectives on the design, the use and the evaluation of Smart Applications for Ambient Intelligence. It congregates works showing the latest advancements in simulated smart environments as well as successful deployments in the real world, with contributions from revised and extended papers from the ISAmI'11 (International Symposium on Ambient Intelligence) event held at the University of Salamanca, Spain in April, 2011.

Ambient Intelligence (AmI) is recognized as an established research field where smart and sensitive devices are used as proactive tools to assist people with their day-to-day activities, making everyone's life more comfortable. The application of software engineering best practices can strengthen the successful development of systems that offer context-aware suggestions or automate tasks according to the current situation of their users.

The papers in this Thematic Issue highlight various best practices for engineering intelligent software architectures, with development methodologies for building intelligent applications and case studies on software requirements and testing of smart behavior.

\section{In this thematic issue}

The Thematic Issue starts with a survey by Preuveneers and Novais about software engineering techniques and best practices for the development of smart applications in Ambient Intelligence. This survey presents insights about the latest developments in this domain and highlights directions to further the research into the successful design, development and evaluation of Ambient Intelligence frameworks and applications.

García-Valverde, Campuzano, Serrano, Villa and Botía present in their paper "Simulation of human behaviors for the validation of ambient intelligence services" a methodology to verify and validate Ambient Intelligence services. From a software engineering perspective, it is very hard to test applications given the user-centric nature of AmI services and the complex behavior of human beings. This work aims to bridge this gap by offering a methodological approach for early validation based on simulation of social models. The added value of this approach is that social simulation can deal with complex social processes that traditional computational and mathematical models have difficulties with.

Reuse is a common practice in software engineering. However, when different initiatives to achieve a common goal divert, building on each other's work may become impractical. Such a case was investigated by Griol, Molina and Callejas in their paper "Bringing together commercial and academic perspectives for the development of intelligent AmI interfaces". In this work, the authors discuss the gap between research and commercial initiatives on spoken dialog systems. While the former is exploring learning dialog systems using statistical models trained with real conversations, the latter has followed a different path using rule-based approaches and Voice$\mathrm{XML}$, an industry-wide standard. The authors present a technique that combines the best of both worlds to reduce the effort required to define optimal dialog strategies and implement these spoken dialog systems. From a software engineering perspective, the authors present an interesting case on how reuse of tools, techniques and platforms from both the academic and commercial worlds has helped them to

${ }^{*}$ Corresponding author. E-mail: davy.preuveneers@cs.kuleuven.be. 
simplify the development of novel intelligent AmI interfaces.

Krüger, Yordanova, Burghardt and Kirste present in their paper "Towards creating assistive software by employing human behavior models" an intent recognition technique for use in assistive software. The advantage of the technique is that it does not require training data to get a good recognition rate for the user's state. The authors generate probabilistic inference systems from causal models for human behavior. From a software engineering point of view, this work is a nice illustration of how gathering an explicit list of requirements upfront can help with creating an adequate software architecture for assistive systems in a flexible way that is robust and easy to adapt to new components in the system and changes in the environment.

An agent-oriented modeling approach for developing open and adaptive societal information systems for decentralized decision-making is the topic of the paper by Taveter, Du and Huhns entitled "Engineering societal information systems by agent-oriented modeling". In such systems, interactions amongst participants are enhanced through software agents to guide their individual actions and decisions, with the aim to achieve effective global behavior. This topic fits well within the scope of this thematic issue as this work provides a good illustration of how a wellknown software engineering paradigm, i.e. agent oriented modeling and multi-agent systems, can be applied for the development of large-scale societal information systems.

Bernini, Fiamberti, Micucci, Tisato discuss in "Architectural abstractions for spaces-based communication in Smart Environments" a set of spaceaware communication primitives and architectural abstractions that support the seamless integration of heterogeneous application components whose cooperation enhances the functionalities of an open-ended smart environment. From a software engineering point of view, this work highlights the crucial role of suitable communication mechanisms (direct communication, tuple mediated communication and eventbased publish/subscribe) for smart environments, and uses software architecture views as a best practice to analyze such environments in depth.

\section{Acknowledgments}

The guest editors wish to thank the many colleagues who have taken their precious time to review the papers for this thematic issue. The thematic issue of the Journal of Ambient Intelligence and Smart Environments would not have been possible without the help and determination of the editorial staff and last but not the least, we would like also to thank all the authors for their hard and highly valuable work. 